\title{
The Localized Inflammatory Response to Bronchoscopic Thermal Vapor Ablation
}

\author{
Daniela Gompelmann ${ }^{\mathrm{a}}$ Ralf Eberhardt ${ }^{\mathrm{a}} \quad$ Armin Ernst $^{\mathrm{e}}$ Peter Hopkins $^{\mathrm{i}}$ \\ Jim Egan ${ }^{k}$ Franz Stanzel $^{\text {b }}$ Arschang Valipourl Manfred Wagner ${ }^{c}$ \\ Christian Witt $^{d}$ Kimberly M. Baker ${ }^{f}$ Mark H. Gotfried ${ }^{g}$ Steven Kesten ${ }^{h}$ \\ Gregory Snell ${ }^{j}$ Felix J.F. Herth ${ }^{a}$
}

\begin{abstract}
apneumology and Critical Care Medicine, Thoraxklinik at University of Heidelberg and Translational Lung Research Center (TLRC), Member of the German Center for Lung Research (DZL), Heidelberg, ${ }^{\text {b } Z e n t r u m ~ f u ̈ r ~ P n e u m o l o g i e, ~}$

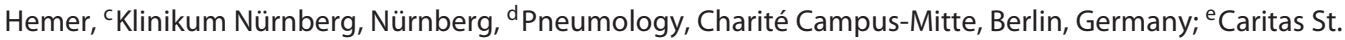
Elizabeth's Medical Center, Boston, Mass., fUniversity of lowa, lowa City, lowa, 9 Pulmonary Associates, Phoenix, Ariz.,

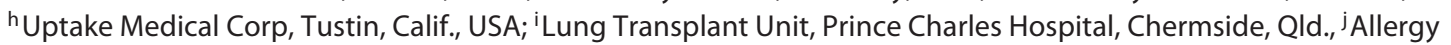

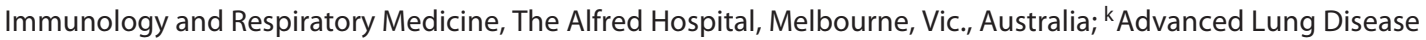
Program, Mater Misericordiae University Hospital, Dublin, Ireland; 'Ludwig-Boltzmann-Institute for COPD, OttoWagner-Hospital, Vienna, Austria
\end{abstract}

\section{Key Words}

Emphysema $\cdot$ Endoscopy $\cdot$ Lung volume reduction

\begin{abstract}
Background: Bronchoscopic thermal vapor ablation (BTVA) reduces lung volumes in emphysema patients by inducing a localized inflammatory response (LIR) leading to a healing process of fibrosis, but may also increase symptoms. Objectives: We sought to evaluate whether the clinical manifestation of LIR correlated with patient outcome. Methods: Respiratory adverse events and inflammatory markers were analyzed from a multicenter trial of BTVA in patients with upper-lobe-predominant emphysema. End points including changes in forced expiratory flow $\left(\mathrm{FEV}_{1}\right)$, lobar volume, St. George's Respiratory Questionnaire (SGRQ), modified Medical Research Council (mMRC) and 6-minute-walk distance (6MWD) were analyzed according to the presence or absence of a respiratory adverse event requiring treatment with an antibiotic or steroid. Results: Forty-four patients received BTVA. Increases of inflammatory markers were observed
\end{abstract}

with a peak between the second and fourth week. Eighteen respiratory adverse events occurred in 16 patients within 30 days of BTVA, requiring antibiotics and/or steroids. These patients had significantly greater lobar volume reduction (65.3 vs. $33.4 \%, p=0.007$ ) and a change in residual volume at 12 months (-933 vs. $13 \mathrm{ml}, \mathrm{p}<0.001)$ associated with a greater improvement of exercise capacity and health-related quality of life than patients without respiratory adverse events. Conclusion: Patients with more prominent respiratory symptoms in the first 30 days following BTVA experience greater efficacy. The clinical manifestations of the LIR are predictive of long-term clinical benefits.

Copyright $\odot 2013$ S. Karger AG, Basel

\section{Introduction}

Endoscopic lung volume reduction presents a new, effective treatment modality for patients with severe chronic obstructive pulmonary disease (COPD) and emphysema. In general, occlusive and nonocclusive techniques

\section{KARGER}

E-Mail karger@karger.com

www.karger.com/res
C 2013 S. Karger AG, Basel

0025-7931/13/0864-0324\$38.00/0
Dr. med. D. Gompelmann

Pneumology and Critical Care Medicine, Thoraxklinik Heidelberg Amalienstrasse 5

DE-69126 Heidelberg (Germany)

E-Mail daniela.gompelmann@ @horaxklinik-heidelberg.de 
can be differentiated [1]. Whereas the occlusive devices can only be used in emphysema patients with low collateral ventilation, the nonocclusive techniques also present a therapeutic option in patients with low- or high-collateral ventilation $[2,3]$.

One of these nonocclusive techniques is bronchoscopic thermal vapor ablation (BTVA, InterVapor ${ }^{\mathrm{TM}}$, Uptake Medical Corporation, Seattle, Wash., USA) offering a therapeutic modality in patients with severe upper-lobepredominant emphysema. The BTVA system delivers thermal energy in the form of heated water vapor to the most emphysematous destroyed areas of the lungs and thus induces a localized inflammatory response (LIR). The treated area heals with fibrosis and scarring, resulting in lung volume reduction [3-5]. Due to decreased hyperinflation in favor of adjacent healthier lung parenchyma and optimized respiratory mechanics, breathlessness is reduced and exercise capacity increases, leading to an improved quality of life $[3,4]$. Therefore, this minimally invasive technique resembles lung volume reduction surgery, but with less morbidity and mortality $[4,6]$. However, the LIR is accompanied by a systemic 'flu-like reaction' with varying symptoms such as cough, sputum, fever, dyspnea and hemoptysis. An elevation of inflammatory markers in the blood and localized chest X-ray changes can also be observed.

We hypothesized that the magnitude of the early, short-term LIR would be associated with the magnitude of the natural healing process of contraction fibrosis and hence the degree of lung volume reduction and clinical response following BTVA. The aim of this study was to examine the association of the LIR and patient outcomes over 1 year following BTVA treatment, by reexamining a previously published clinical trial $[4,7]$.

\section{Materials and Methods}

Nonspecific inflammatory markers and lower respiratory adverse events were analyzed from 2 multicenter, single-arm trials of BTVA treatment in patients with severe upper-lobe-predominant emphysema, that were performed in Europe, the USA and Australia $[4,7]$. The protocol of these virtually identical prospective trials (NCT 01041586 and NCT 01102712) was approved by institutional review boards or ethics committees. After written informed consent, all patients underwent BTVA, which consists of the delivery of heated water vapor in the most emphysematous, destroyed, targeted lobe in a single bronchoscopic setting.

\section{Patient Inclusion Criteria}

The inclusion criteria of the patients have been described previously [4]. They can be briefly summarized as follows: severe upperlobe-predominant emphysema documented by thin-section non- enhanced multislice computed tomography, forced expiratory volume in $1 \mathrm{~s}\left(\mathrm{FEV}_{1}\right) 15-45 \%$ predicted, residual volume (RV) $>150 \%$ predicted and total lung capacity (TLC) $>100 \%$ predicted. BTVA was performed with a target vapor dose of $10 \mathrm{cal} / \mathrm{g}$ in all patients in either the right upper lobe or left upper lobe, excluding the lingula $[3,4]$.

\section{Follow-Up and End Points}

All patients were hospitalized for a minimum of $24 \mathrm{~h}$ following BTVA for monitoring. Follow-up clinic visits were scheduled at 1 , 2, 12, 26 and 52 weeks posttreatment. End points included $\mathrm{FEV}_{1}$, health-related quality of life documented by the St. George's Respiratory Questionnaire (SGRQ), the modified Medical Research Council (mMRC) dyspnea scale, 6-minute-walk distance (6MWD), lung volume reduction by quantitative analysis of CT as well as adverse events. Lobar volume was calculated quantitatively from the preinterventional high-resolution computed tomography (HRCT) using PW2 software (VIDA Diagnostics, Iowa City, Iowa, USA).

\section{LIR Assessment}

During clinic visits, blood samples were taken for analysis of inflammatory markers including white blood cells (WBCs), neutrophils, C-reactive protein (CRP) and erythrocyte sedimentation rate (ESR). In addition, chest X-rays were performed at clinic visits for assessment of abnormalities in the treated area.

At each clinic visit, investigators were asked to record the presence of any and all adverse events. An adverse event was defined prospectively as any undesired, noxious or pathological change as indicated by signs, symptoms or laboratory changes that occur during the study, whether or not considered to be related to the study device or protocol. For the purposes of our analysis, a lower respiratory adverse event was included if the investigator used a lower respiratory diagnostic term (i.e. acute bronchitis, exacerbation, pneumonia or lower respiratory tract infection) and prescribed antibiotics and/or steroids for treatment of the event. Furthermore, the analysis is focused on those events that occurred within 30 days of treatment with BTVA.

\section{Results}

\section{Clinical Outcome}

From December 2009 to December 2010, 44 patients with severe heterogeneous upper-lobe-predominant emphysema were enrolled and received BTVA. BTVA was administered to the right upper lobe in 24 patients and to the left upper lobe in 20 patients. Clinical efficacy outcomes $[4,7]$ were as follows [mean (SD) improvements at 6 and 12 months]: $\mathrm{FEV}_{1} 141$ (166) and 86 (174) ml, FVC 271 (455) and 249 (429) $\mathrm{ml}, \mathrm{RV}-406$ (714) and -303 (776) $\mathrm{ml}$, SGRQ total score -14 (15) and -11 (14) units, mMRC -0.9 (1) and -0.8 (1) and 6-MWD 47 (67) and 19 (64) meters ( $\mathrm{p}<0.001$ for all except for $6-\mathrm{MWD}$ at 12 months). The BODE score improved 1.5 (1.8) and 1.3 (1.8) units at 6 and 12 months, respectively ( $p<0.001$ for both) [4, 7]. 
Table 1. Changes (mean \pm SD) in inflammatory markers over 6 months

\begin{tabular}{|c|c|c|c|c|c|}
\hline & $\Delta$ Week 1 & $\Delta$ Week 2 & $\Delta$ Week 4 & $\Delta$ Month 3 & $\Delta$ Month 6 \\
\hline \multicolumn{6}{|c|}{ Following treatment with BTVA $[4]$} \\
\hline WBCs, $10^{9} / 1$ & $1.51 \pm 2.56$ & $3.53 \pm 3.42$ & $2.60 \pm 4.00$ & $1.28 \pm 2.86$ & $0.62 \pm 2.47$ \\
\hline Neutrophils, $10^{9} / 1$ & $1.42 \pm 2.14$ & $3.36 \pm 3.26$ & $2.35 \pm 3.86$ & $1.05 \pm 2.23$ & $0.42 \pm 2.66$ \\
\hline $\mathrm{ESR}, \mathrm{mm} / \mathrm{h}$ & $15.3 \pm 11.3$ & $22.8 \pm 23.9$ & $27.0 \pm 28.7$ & $7.11 \pm 14.36$ & $4.50 \pm 14.6$ \\
\hline $\mathrm{CRP}, \mathrm{mg} / \mathrm{dl}$ & $1.70 \pm 4.78$ & $8.10 \pm 21.2$ & $9.71 \pm 38.4$ & $1.11 \pm 5.07$ & $0.67 \pm 2.78$ \\
\hline \multicolumn{6}{|c|}{ In patients with and without lower respiratory adverse events following treatment with BTVA } \\
\hline \multicolumn{6}{|c|}{ Patients with lower respiratory adverse event $(\mathrm{n}=16)$} \\
\hline WBCs, $10^{9} / 1$ & $2.68 \pm 3.9$ & $5.02 \pm 4.5$ & $5.43 \pm 4.59$ & $2.19 \pm 3.34$ & $1.39 \pm 3.31$ \\
\hline Neutrophils, $10^{9} / 1$ & $2.52 \pm 4.19$ & $5.16 \pm 4.33$ & $5.16 \pm 4.62$ & $-0.85 \pm 6.7$ & $0.99 \pm 3.98$ \\
\hline $\mathrm{ESR}, \mathrm{mm} / \mathrm{h}$ & $11.5 \pm 21.8$ & $29.44 \pm 32.78$ & $54.1 \pm 29.81$ & $9.33 \pm 14.4$ & $3.0 \pm 10.9$ \\
\hline $\mathrm{CRP}, \mathrm{mg} / \mathrm{dl}$ & $3.32 \pm 7.13$ & $6.35 \pm 33.59$ & $25.69 \pm 61.48$ & $2.91 \pm 8.6$ & $0.29 \pm 1.37$ \\
\hline \multicolumn{6}{|c|}{ Patients without lower respiratory adverse event $(n=28)$} \\
\hline WBCs, $10^{9} / 1$ & $0.93 \pm 1.27$ & $2.78 \pm 2.50$ & $1.08 \pm 2.83$ & $0.82 \pm 2.55$ & $0.21 \pm 1.82$ \\
\hline Neutrophils, $10^{9} / 1$ & $0.80 \pm 1.16$ & $2.36 \pm 1.96$ & $0.71 \pm 2.05$ & $0.43 \pm 1.03$ & $0.07 \pm 1.54$ \\
\hline $\mathrm{ESR}, \mathrm{mm} / \mathrm{h}$ & $11.15 \pm 8.36$ & $19.29 \pm 17.7$ & $12.68 \pm 14.44$ & $6.0 \pm 14.6$ & $5.57 \pm 17.5$ \\
\hline $\mathrm{CRP}, \mathrm{mg} / \mathrm{dl}$ & $0.96 \pm 3.08$ & $2.0 \pm 3.46$ & $0.50 \pm 1.21$ & $0.21 \pm 0.93$ & $0.87 \pm 3.27$ \\
\hline
\end{tabular}

\section{Local Inflammatory Response}

As stated previously, a total of 18 lower respiratory tract events requiring treatment with antibiotics and/or steroids were observed in 16 patients within 30 days of BTVA [4]. The inflammatory reaction was frequently associated with clinical symptoms such as fever, cough, sputum, dyspnea or mild hemoptysis. Clinically, symptoms, when present, peaked within the first 2-4 weeks and resolved within 8-12 weeks following BTVA. Vital signs measured at follow-up clinic visits showed no overall change from baseline. All chest X-ray reports were reviewed. A total of $81 \%$ of patients had chest X-ray reports within $48 \mathrm{~h}$ of BTVA noting opacification in the treated area, indicative of a LIR.

The mean changes in inflammatory markers are shown in table 1. Patients who experienced a respiratory event developed a higher increase of the inflammatory parameters (fig. 1). Antibiotics alone were prescribed for 7 of the 16 patients, steroids alone were prescribed for 3 , and 6 patients were prescribed antibiotics and steroids. Ten patients who experienced lower respiratory adverse events were hospitalized for observation and therapy; 1 developed increased symptoms after discharge and died in a hospice 67 days after treatment with a diagnosis of endstage emphysema.

\section{Association between LIR and Efficacy Outcome}

Patients with a lower respiratory event reported within 30 days of treatment had greater improvements in efficacy outcomes than patients without events in the first 30 days following BTVA. Significant differences were observed in lobar volume and residual volume at 3 months $(-70.4$ vs. $-37.0 \%, \mathrm{p}<0.001$ and -741 vs. $-255 \mathrm{ml}, \mathrm{p}=0.012)$, 6 months $(-68.7$ vs. $-35.4 \%, \mathrm{p}=0.001$ and -931 vs. $-123 \mathrm{ml}, \mathrm{p}<0.001$ ) and 12 months ( -65.3 vs. $-33.4 \%, \mathrm{p}=$ 0.007 and -933 vs. $13 \mathrm{ml}, \mathrm{p}<0.001)$ as well as in the $\mathrm{FEV}_{1}$ at 3 months (216 vs. $101 \mathrm{ml}, \mathrm{p}=0.04$ ). The lobar volume reduction, changes in $R V, F E V_{1}, 6-M W D, m M R C$ and SQRG in patients with and without a lower respiratory adverse event are shown in table 2 and figure 2.

\section{Association between LIR and Targeted Lung Volume}

An additional post hoc analysis was conducted on the subgroup of 10 patients who were hospitalized for a lower respiratory tract event within 30 days of treatment, in order to determine if a risk factor for a more severe event could be identified. Patients with a high treated lobar volume appeared to be at a higher risk of hospitalizations in the first 30 days. In total, $12 / 44$ patients had a target lobar volume $>1,700 \mathrm{ml}$ and the other $32 \mathrm{had}$ a target volume $<1,700 \mathrm{ml}$. Of the 12 higher-volume patients, 7 (58.3\%) were hospitalized for a lower respiratory adverse event whereas only $3 / 32$ patients $(9.4 \%)$ with a lower targeted lobe volume were hospitalized (fig. 3). In terms of the lower respiratory events $(n=10)$ occurring in these hospitalized patients, 7 (70\%) occurred in those with high volumes and $3(30 \%)$ in those with lower volumes. The efficacy outcome measures of both groups are presented in table 3 and indicate that the patients with lobar volumes $>1,700 \mathrm{ml}$ had higher efficacy as observed by lobar 

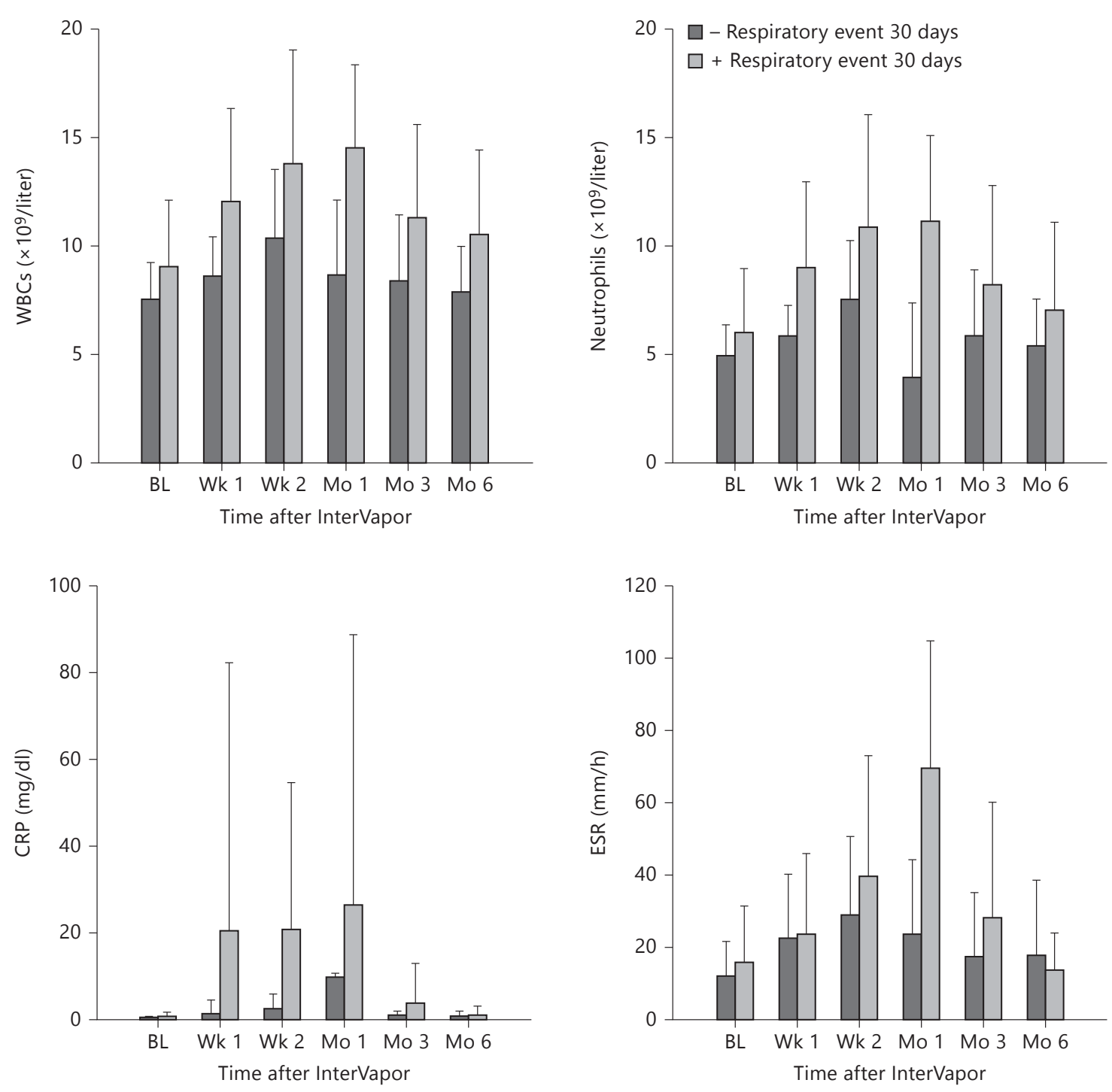

Fig. 1. Inflammatory markers (mean $\pm \mathrm{SD}$ ) over time in patients with and without lower respiratory adverse event within 30 days of BTVA. BL = Baseline; Mo = month; $\mathrm{Wk}=$ week.

volume reduction at $3(\mathrm{p}=0.004), 6(\mathrm{p}=0.009)$ and 12 months $(\mathrm{p}=0.025)$, in RV at $3(\mathrm{p}<0.001)$ and 6 months $(\mathrm{p}=0.003)$ as well as in $\operatorname{FEV}_{1}(\mathrm{p}=0.024)$ and 6-MWD $(\mathrm{p}=0.033)$ at 6 months after BTVA treatment.

\section{Discussion}

BTVA represents an effective treatment option for patients with upper-lobe-predominant emphysema. It resembles lung volume reduction surgery in that lung tissue is ablated, but with the advantage of a minimally invasive approach. Lung volume reduction is achieved with BTVA by delivering thermal energy in the form of heated water vapor through a disposable bronchoscopic catheter that leads to contraction fibrosis of targeted lung compartments $[4,5,7]$. Following BTVA in the described clinical trial, patients experienced improvements in airflow, lung volumes, exercise capacity and health-related quality of life that were present for the 12 months of follow-up [4, 7]. BTVA has a well-understood safety profile that is related to the mechanism of action resulting in the clinical 
Table 2. Changes (mean $\pm \mathrm{SD}$ ) in lobar volume, $\mathrm{RV}, \mathrm{FEV}_{1}, 6-\mathrm{MWD}, \mathrm{mMRC}$ and SGRQ total score in patients with and without lower respiratory adverse events within 30 days of BTVA treatment

\begin{tabular}{|c|c|c|c|c|c|c|}
\hline & \multicolumn{3}{|c|}{ Respiratory event $(\mathrm{n}=16)$} & \multicolumn{3}{|c|}{ No respiratory event $(\mathrm{n}=28)$} \\
\hline & 3 months & 6 months & 12 months & 3 months & 6 months & 12 months \\
\hline LoV, \% & $-70.4 \pm 26.3^{*}$ & $-68.7 \pm 26.8^{*}$ & $-65.3 \pm 28.5^{*}$ & $-37.0 \pm 29.0$ & $-35.4 \pm 29.9$ & $-33.4 \pm 28.2$ \\
\hline $\mathrm{LoV}, \mathrm{ml}$ & $-1,225$ & $-1,195$ & $-1,214.9$ & -478 & -450 & -452.2 \\
\hline $\mathrm{RV}, \mathrm{ml}$ & $-741 \pm 554^{*}$ & $-931 \pm 629 *$ & $-933 \pm 623^{*}$ & $-255 \pm 573$ & $-123 \pm 593$ & $13 \pm 647$ \\
\hline $\mathrm{FEV}_{1}, \mathrm{ml}$ & $216 \pm 209^{*}$ & $189 \pm 192$ & $166 \pm 131$ & $101 \pm 146$ & $115 \pm 148$ & $48 \pm 181$ \\
\hline $\mathrm{FEV}_{1}, \% \Delta$ & $25.0 \pm 23.0$ & $23.0 \pm 23.4$ & $19.0 \pm 14.0$ & $12.8 \pm 19.3$ & $13.5 \pm 17.8$ & $6.1 \pm 21.8$ \\
\hline mMRC & $-1.1 \pm 0.7$ & $-1.3 \pm 0.9$ & $-1.1 \pm 0.8$ & $-0.6 \pm 1.2$ & $-0.7 \pm 1.1$ & $-0.7 \pm 1.0$ \\
\hline 6-MWD, m & $32.8 \pm 79.9$ & $73.0 \pm 68.8$ & $38.5 \pm 81.0$ & $19.4 \pm 58.2$ & $32.2 \pm 62.9$ & $9.3 \pm 53.5$ \\
\hline SGRQ, patients & $-12.4 \pm 16.5$ & $-15.1 \pm 19.0$ & $-12.2 \pm 17.0$ & $-10.4 \pm 14.7$ & $-13.3 \pm 13.0$ & $-10.5 \pm 12.6$ \\
\hline
\end{tabular}

$* \mathrm{p}<0.05$ compared to patients with no respiratory event. LoV $=$ Lobar volume.

benefits $[4,7,8]$. The application of thermal energy to tissue leads to an early and short-term inflammatory response that appears clinically as an exacerbation of COPD. The reaction appears to peak within the first 2-4 weeks and resolves over 8-12 weeks.

Symptoms from the LIR have already been reported in the earliest human study reported 2009 by Snell et al. [8]. In this prospective trial, 11 patients with severe upper-lobe-predominant emphysema underwent unilateral BTVA at a lower dose of approximately $5 \mathrm{cal} / \mathrm{g}$. In 10 of the 11 patients, symptoms such as cough, mild-tomoderate hemoptysis, fatigue and nausea could be observed following BTVA. A total of 7 COPD exacerbations occurred in 4 patients, whereby 3 were judged infectious and 4 noninfectious. Furthermore, 2 cases of pneumonitis within the first week following the treatment were reported. In 2 patients with COPD exacerbation and 1 patient with pneumonitis, hospitalization was required. All of these adverse events resolved without sequelae.

In our trial, at the higher-energy dose of $10 \mathrm{cal} / \mathrm{g}$ that was delivered, 16 of 44 patients (36.4\%) experienced 18 lower respiratory tract events indicative of a LIR within 30 days of BTVA. These LIRs were given different diagnostic terms (although all consistent with lower respiratory tract events) and were judged by the investigator as needing treatment with antibiotics and/or corticosteroids. In reality, it is not clear whether infection actually does play a part; however, clinically, it is often simplest to assume the possibility and treat for infection presumptively.

Patients with these LIRs experienced a greater increase in nonspecific inflammatory markers than the pa- tients without a lower respiratory adverse event. This finding indicates that the thermally induced inflammatory reaction begins in the treated area of the lung but then results in a systemic inflammation with elevation of leukocytes, CRP, neutrophils and ESR associated with respiratory symptoms. It is most likely that the systemic effect represents spillover from the lung rather than a primary systemic effect. Clinical manifestation as well as elevated inflammatory markers peaked within the first 2-4 weeks and resolved within 8-12 weeks following BTVA. Due to the very similar time course of the different inflammatory markers in this trial, the impact on the efficacy of the early-rising inflammation markers, e.g. CRP and the late-rising markers e.g. ESR was not differentiated.

It appears that the LIR is essential for the desired lobar volume reduction. The LIR initiates the healing process that is associated with peribronchial and parenchymal fibrosis formation replacing diseased lung tissue [5]. In this trial, efficacy outcomes measures showed that patients who had a lower respiratory tract event reported within 30 days of treatment experienced greater improvements than patients without events in the first 30 days following BTVA. In particular, the patients with a targeted lung volume $>1,700 \mathrm{ml}$ experienced substantial clinical efficacy on multiple outcome variables following a lower respiratory tract adverse event.

The LIR is also presumably responsible for an increased risk for symptoms that may require pharmacotherapy and hospitalization. As a practical recommendation, all patients treated with BTVA require careful postinterventional monitoring over the first month. Prophylactic administration of antibiotics has been in- 

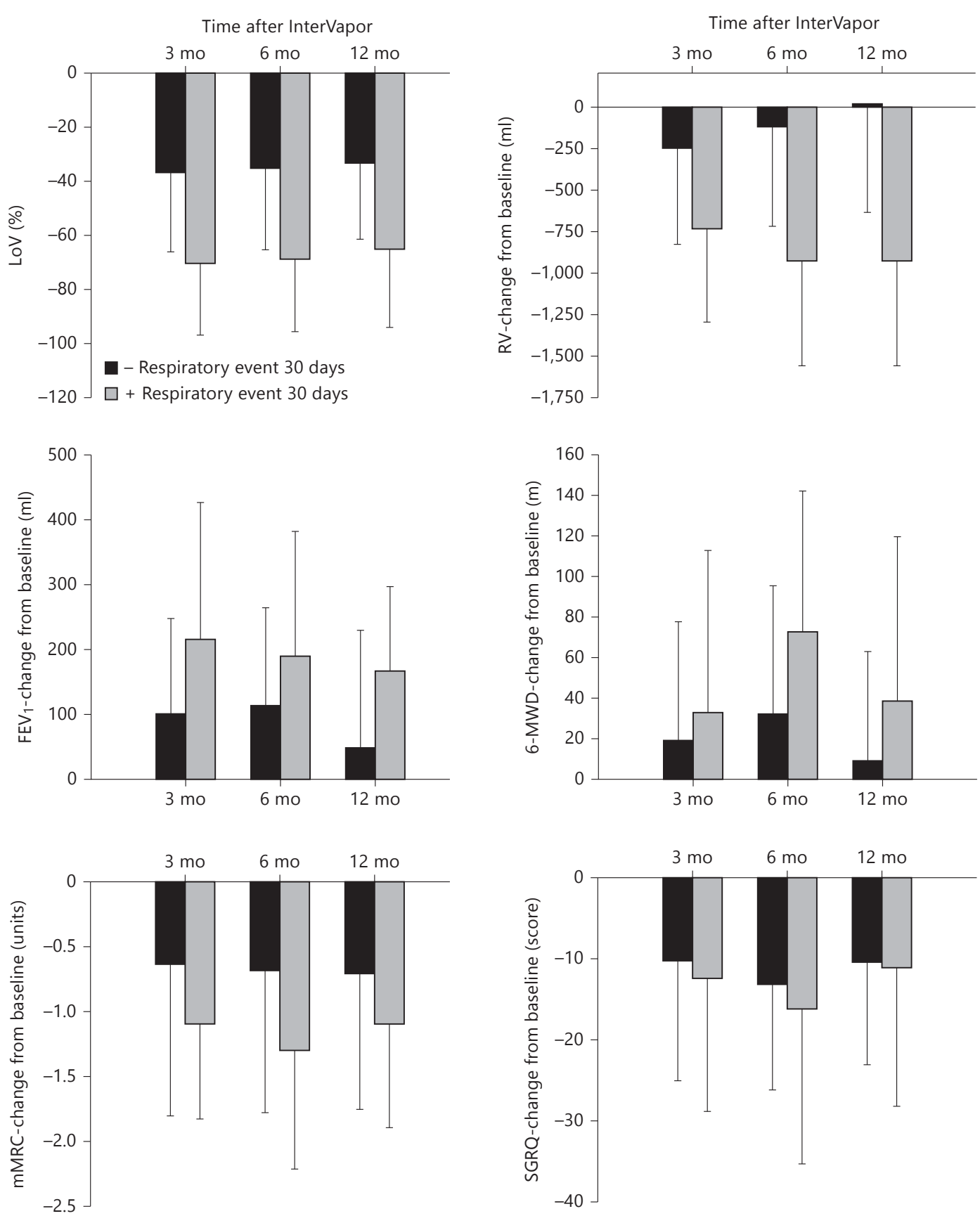

Fig. 2. Changes (mean $\pm \mathrm{SD}$ ) in lobar volume (LoV), $\mathrm{FEV}_{1}, 6-\mathrm{MWD}, \mathrm{mMRC}$ and SGRQ total score over time in patients with and without a lower respiratory tract event within 30 days of BTVA. mo $=$ Months.

corporated into the treatment protocol (on the day of BTVA and for at least 10 days afterwards) based on the previously conducted clinical trial $[4,7]$. A somewhatrelated concern with BTVA would be the impact of systemic steroids. It might be hypothesized that steroids would decrease the healing response such that effective lung volume reduction would not occur. However, our analysis indicates that significant efficacy can be expected despite systemic steroids in patients with sufficient symptoms warranting treatment with systemic anti-inflamma- 


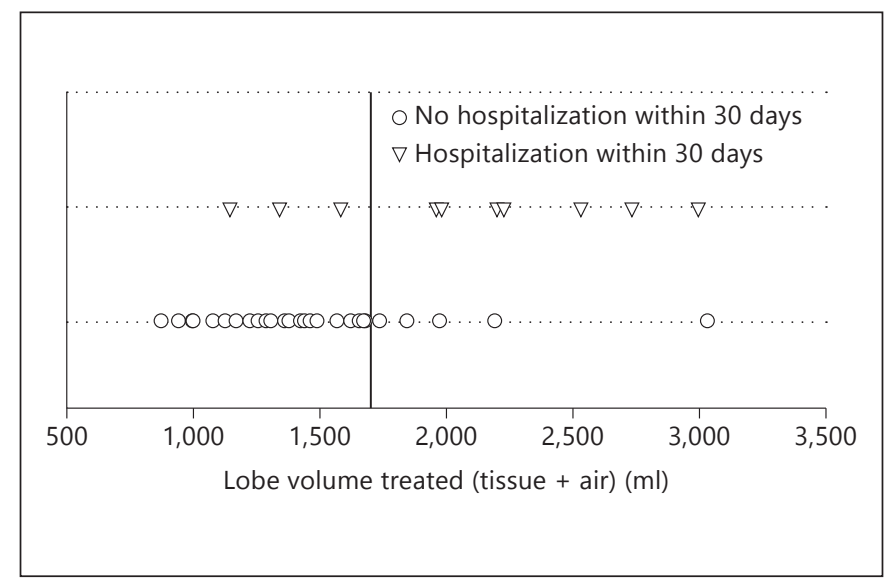

Fig. 3. Hospitalization due to respiratory adverse event in patients with targeted lobar volume $>1,700 \mathrm{ml}$ and $<1,700 \mathrm{ml}$.

Table 3. Efficacy outcome (mean \pm SD) of patients with targeted lobe volume $>1,700 \mathrm{ml}$ and $<1,700 \mathrm{ml}$ following BTVA

\begin{tabular}{lccc}
\hline & 3 months & 6 months & 12 months \\
\hline$>1,700 \mathrm{ml}(\mathrm{n}=12)$ & & & \\
$\quad$ Lobar volume, \% & $-73.1 \pm 27.8^{*}$ & $-70.2 \pm 30.0^{*}$ & $-65.3 \pm 33.2^{*}$ \\
RV, ml & $-845 \pm 623^{*}$ & $-964 \pm 687^{*}$ & $-699 \pm 1,032$ \\
FEV, ml & $220 \pm 236$ & $242 \pm 212^{*}$ & $122 \pm 190$ \\
SGRQ, units & $-4.9 \pm 14.7$ & $-13.2 \pm 15.9$ & $-12.8 \pm 16.6$ \\
6-MWD, m & $16.6 \pm 52.4$ & $85.3 \pm 55.6^{*}$ & $40.4 \pm 91.2$ \\
$<1,700 \mathrm{ml}(\mathrm{n}=32)$ & & & \\
Lobar volume, \% & $-40.3 \pm 29.5$ & $-39.5 \pm 30.3$ & $-36.8 \pm 27.8$ \\
RV, ml & $-283 \pm 542$ & $-220 \pm 629$ & $-150 \pm 610$ \\
FEV, ml & $114 \pm 148$ & $107 \pm 136$ & $73 \pm 169$ \\
SGRQ, units & $-13.0 \pm 15.0$ & $-14.2 \pm 15.1$ & $-10.4 \pm 13.1$ \\
6-MWD, m & $25.6 \pm 68.8$ & $33.6 \pm 66.4$ & $12.0 \pm 53.6$ \\
\hline
\end{tabular}

$* \mathrm{p}<0.05$ compared to patients $<1,700 \mathrm{ml}$.

tory therapy. It is not known whether prophylactic administration of systemic steroids would decrease the severity of symptomatic inflammatory responses without compromising efficacy.

Previously, it was unclear why the extent of the LIR varied among patients following BTVA. Therefore, predictive factors were sought that would presumably lead to an improved approach and an enhanced favorable benefit-risk profile. One of these factors seems to be the targeted lung volume, which is related to the total vapor dose. In this prospective trial, a higher rate of hospitalization due to lower respiratory events was ob- served in patients with a targeted lobar volume $>1,700$ $\mathrm{ml}$. Although these patients experienced a better outcome following BTVA, this observation suggests that patients with higher volumes may require increased vigilance in monitoring or an alteration in the treatment approach.

The main limitation of this trial is its retrospective design. This analysis incorporates data collected for the previously published prospective multicenter trial summary of two protocols [4]. The occurrence of serious and nonserious adverse events was the only primary end point in the smaller 10-patient study protocol. In the other 34-patient protocol, the quality characteristics of the LIR (clinical features and analysis of inflammatory markers) were not among the main end points. Furthermore, only leukocytes, neutrophils, CRP and ESR values were obtained, but no further inflammation markers like fibrinogen, cytokines or procalcitonin were collected.

Another limitation is that the definition of respiratory adverse events was based only on a combination of clinical symptoms, laboratory results and the need of antibiotics and/or steroids, and is thus not necessarily reproducible. Being a retrospective, multicenter study, medical strategy for respiratory adverse event was not always uniform for all patients.

The aim of this trial is to draw attention to the inflammatory respiratory events following BTVA and to describe its management and its association with efficacy. In Europe and Australia, but not in the USA, BTVA has been approved and has received the CE mark. However, to date, our knowledge of BTVA is based on a limited number of patients, so it should be performed under research protocol. Currently, studies are underway in Europe and Australia with a focus on patient selection and on finding the balance between efficacy and safety.

In summary, BTVA represents an effective therapeutic option for patients with upper-lobe-predominant, severe emphysema. The treatment is associated with a LIR that can be characterized by clinical, laboratory and radiologic findings. Such findings appear to peak within the first 2-4 weeks and resolve by 3 months. Patients with more prominent respiratory symptoms in the first 4 weeks have greater long-term efficacy, with the risk for hospitalization appearing to be related to the absolute targeted lung volume. As with all of the endoscopic approaches, further analyses of databases should continue in order to refine the therapeutic approaches that focus on patient profiles and phenotypes. 


\section{References}

1 Herth FJ, Gompelmann D, Ernst A, Eberhardt R: Endoscopic lung volume reduction. Respiration 2010;79:5-13.

-2 Sciurba FC, Ernst A, Herth FJ, Strange C, Criner GJ, Marquette CH, Kovitz KL, Chiacchierini RP, Goldin J, McLennan G: A randomized study of endobronchial valves for advanced emphysema. N Engl J Med 2010;363:1233-1244.

-3 Gompelmann D, Heussel CP, Eberhardt R, Snell G, Hopkins P, Baker K, Witt C, Valipour A, Wagner M, Stanzel F, Egan J, Ernst A, Kesten S, Herth FJ: Efficacy of bronchoscopic thermal vapor ablation and lobar fissure completeness in patients with heterogeneous emphysema. Respiration 2012;83:400-406.
4 Snell G, Herth FJ, Hopkins P, Baker KM, Witt C, Gotfried MH, Valipour A, Wagner M, Stanzel F, Egan J, Kesten S, Ernst A: Bronchoscopic thermal vapor ablation therapy in the management of heterogeneous emphysema. Eur Respir J 2012;39:1326-1333.

$>5$ Tuck SA, Lopes-Berkas V, Beam S, Anderson JC: Bronchoscopic thermal vapor ablation in a canine model of emphysema. Int J Chron Obstruct Pulmon Dis 2012;7:21-31.

66 Fishman A, Martinez F, Naunheim K, Piantadosi S, Wise R, Ries A, Weinmann G, Wood DE; National Emphysema Treatment Trial Research Group: A randomized trial comparing lung-volume-reduction surgery with medical therapy for severe emphysema. $\mathrm{N}$ Engl J Med 2003;348:2059-2073.
Herth FJF, Ernst A, Baker KM, Egan JJ, Gotfried MH, Hopkins P, Stanzel F, Valipour A, Wagner M, Witt C, Kesten S, Snell $\mathrm{G}$ : Characterization of outcomes one year after endoscopic thermal vapor ablation for patients with heterogeneous emphysema. Int J Chron Obstruct Pulmon Dis 2012;7: 397-405.

-8 Snell GI, Hopkins P, Westall G, Holswoth L, Carle A, Williams TJ: A feasibility and safety study of bronchoscopic thermal vapor ablation: a novel emphysema therapy. Ann Thorax Surg 2009;88:1993-1998. 